\title{
Comparison of initial computed tomography-based target delineation and subsequent magnetic resonance imaging-based target delineation for cervical cancer brachytherapy
}

\author{
Taylor J. Corriher, BS, Sunil W. Dutta, MD, Clayton E. Alonso, MD, Bruce Libby, PhD, Kara D. Romano, MD, \\ Timothy N. Showalter, MD, MPH \\ Department of Radiation Oncology, University of Virginia, Charlottesville, Virginia, USA
}

\begin{abstract}
Purpose: For cervical brachytherapy planning, magnetic resonance imaging (MRI) is preferable to computed tomography (CT) for target delineation. However, due to logistical and financial restrictions, in-room MRI is sometimes not routinely available in brachytherapy centers. Our institution has created a workflow that integrates MRI-based target delineation with an in-room CT scanner, with the aim of improving target coverage and conformality. This study reports the initial dosimetric results with using this workflow.

Material and methods: A retrospective review was performed on 46 consecutive patients who received definitive chemoradiation with 5 fraction intracavitary high-dose-rate (HDR) brachytherapy for cervical cancer. Fraction 1 was planned from CT only. Outpatient MRI was obtained after Smit sleeve placement and first insertion to assess concurrent chemoradiotherapy tumor response. This MRI was registered to the CT for planning fractions 2-5. The median prescription dose for the cohort was 25 Gy (range, 25-29 Gy).

Results: The $\mathrm{D}_{90}$ to the high-risk clinical target volume (HR-CTV) and $\mathrm{D}_{2 \mathrm{cc}}$ rectal dose were increased from fraction 1 to fraction $2-5$ averaged $(p<0.05)$. Among the 18 patients with complete volumetric data, there was no significant difference in HR-CTV size, with an average decrease of $1.73 \mathrm{cc}(p>0.05)$ with MRI fusion. Eleven out of 18 patients had changes in high-risk target volume greater than $20 \%$, with an absolute average change in volume of $31.5 \%$.

Conclusions: The use of asynchronous MRI for target delineation, with co-registration to CT for each fraction of brachytherapy was associated with higher $\mathrm{D}_{90}$ to the HR-CTV. We observed slightly higher $\mathrm{D}_{2 \mathrm{cc}}$ rectal doses with MRI, but cumulative rectal doses were within accepted thresholds. High-risk target volumes were not consistently increased or decreased, but MRI fusion was associated with target volume changes greater than $20 \%$ in over half of the treated patients.
\end{abstract}

J Contemp Brachytherapy 2020; 12, 3: 279-282 DOI: https://doi.org/10.5114/jcb.2020.96871

Key words: brachytherapy, magnetic resonance imaging, uterine cervical neoplasms.

\section{Purpose}

For cervical brachytherapy planning, magnetic resonance imaging (MRI) is preferable to computed tomography (CT) for target delineation [1]. The advantages to MRI-guided brachytherapy are numerous, including excellent soft tissue contrast between residual tumor, cervix, and organs at risk (OARs) [2]. However, due to logistical and financial restrictions, in-room MRI is sometimes not routinely available in brachytherapy centers [3]. Our institution has created a workflow that integrates MRIbased target delineation with an in-room CT scanner, with the aim of improving target coverage and confor- mality [4]. This study reports the initial dosimetric results with using this workflow.

\section{Material and methods}

Institutional review board approval was obtained for the entirety of this study.

\section{Technique}

Our method has been previously described and summarized in Figure 1 [4]. In summary, as of 2014, we performed an outpatient MRI after the first fraction of 
Initial fraction with Smit sleeve placement and CT-guidance

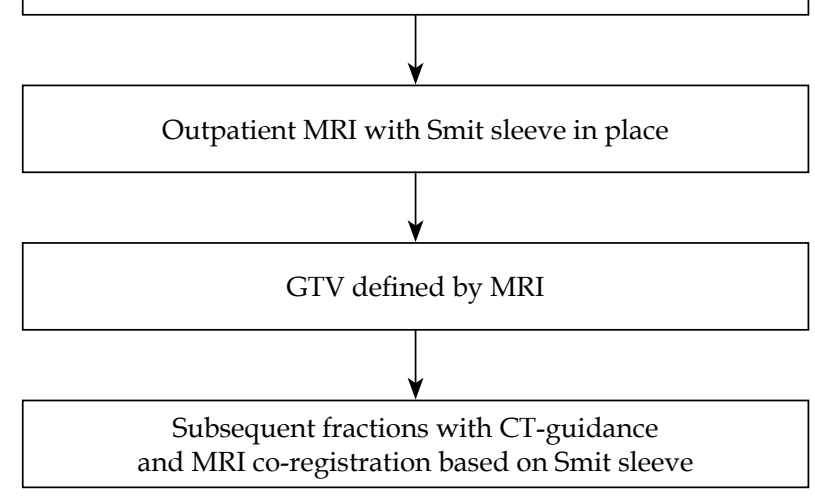

Fig. 1. Institutional workflow. The Smit sleeve is placed under anesthesia. The MRI between the first and subsequent fractions is performed outpatient, separate from brachytherapy procedure, and is also used for external beam response assessment

CT - computed tomography, GTV - gross tumor volume, MRI - magnetic resonance imaging

brachytherapy for primarily two reasons: 1 . To assess response to chemoradiation, and 2. Target volume assessment for brachytherapy planning. The first fraction is entirely CT-based, under general anesthesia, and a Smit sleeve is placed. Prior to subsequent brachytherapy fractions, an outpatient MRI (multiplanar multisequence, T2-weighted) is performed. For each subsequent tandem and ovoid insertion, the MRI was rigidly co-registered to the CT based on the Smit sleeve. The high-risk clinical target volume (HR-CTV) is defined as the entire cervix, cervix tumor as defined by the CT for the first fraction, and MRI registration for fractions 2-5. Organs at risk are contoured on the CT dataset for each fraction and include bladder, rectum, and sigmoid for all patients. No deformable registration techniques were performed.

\section{Analysis}

In this analysis, dosimetry from the first fraction is compared to the average of subsequent fractions using a matched, two-tailed, paired $t$-test. Average, minimum, maximum, and standard deviation of dosimetry changes were calculated. Patients were excluded if they did not receive concurrent sensitizing cisplatin or if a modified fractionation regimen (e.g. 3-4 total brachytherapy fractions) was utilized, as their tumor response may differ. The SPSS Statistics software package (version 24, IBM Corporation, Armonk, New York, NY, USA) was used for all analyses.

\section{Results}

\section{Patient, disease, and treatment characteristics}

Forty-six consecutive patients, treated between September, 2014 and June, 2017, were identified who received definitive chemoradiation with 5 fraction intracavitary HDR brachytherapy for cervical cancer with MRI fusion utilized for fractions 2-5. Median age at time of brachytherapy was 49.9 years old (range, 31.6-71.4 years old). The majority were squamous cell histology (82.6\%), FIGO stage (2009) IB2 or IIB $(25.5 \%$ and $39.2 \%$, respectively), and staged with an MRI $(60.9 \%)$ and positron emission tomography (PET)/CT (89.1\%). Table 1 presents patient, disease, and treatment characteristics.

\section{Dosimetry with or without MRI}

The $\mathrm{D}_{90}$ to the HR-CTV was increased from fraction 1 to fraction 2-5 averaged for individual patients using a match pair analysis (6.07 Gy vs. $6.64 \mathrm{~Gy}$, respectively; $p=0.017$ ). The range in change of $\mathrm{D}_{90}$ was -5.41 to $3.75 \mathrm{~Gy}$, with $31 / 46(67.4 \%)$ experiencing an increase. The $D_{2 c c}$ to rectum was also increased from fraction 1 to fraction 2-5 averaged (2.96 Gy vs. $3.17 \mathrm{~Gy}$, respectively; $p=0.01$ ). The range in change of $\mathrm{D}_{2 \mathrm{cc}}$ to rectum was -1.28 to $1.63 \mathrm{~Gy}$, with 32/46 (69.6\%) experiencing an increase. There was no significant difference in dose to the bladder or sigmoid with respect to MRI registration for the individual patient, as shown in Table 2. Figure 2 represents the average dose to structures by fraction.

In order to quantify the impact on HR-CTV volumes, we measured the percent change from CT to MRI-based target delineation for the eighteen patients, with complete volumetric data. As shown in Figure 3, $11 / 18(61.1 \%)$ were found to have a greater than $20 \%$ change in HR-CTV (6 decreased and 5 increased). The mean absolute HR-CTV change was $7.37 \mathrm{cc}$ (standard deviation, $7.25 \mathrm{cc}$ ), and the range of volume changes in HR-CTV was $-53.09 \%$ to $130.05 \%$.

\section{Discussion}

While no change in overall target volume was found, the current study suggests that physicians are making individualized target changes to prevent either over- or under-coverage, based on the MRI fusion. MRI fusion resulted in higher dose to the HR-CTV across patients, although at the cost of higher rectal doses, which were still within recommended constraints. The higher rectal doses observed may be the result of more aggressive HR-CTV coverage mandated by the MRI-defined gross residual disease. Importantly, this approach is applicable to centers without MRI availability within the department.

Brachytherapy is a critical component of definitive therapy for cervical cancer and should be made available to all patients [5]. GEC-ESTRO promotes adaptive brachytherapy, using a volume-based approach for each fraction [6]. MRI for each fraction with applicators in place is ideal; however, MRI availability is not always readily accessible at most centers. An alternative is using MRI for the first fraction and CT for subsequent fractions. Viswanathan et al. have shown CT to have significant differences in $\mathrm{D}_{90}$ and volume treated with prescription dose compared to MRI-based planning [7]. Our method is particularly attractive because it allows centers to obtain an MRI on an outpatient basis that can by itself result in meaningful volume adjustments in the majority of patients. This study is particularly interesting that the HR-CTV volume can both be over- and under-estimated with CT. 
Table 1. Patient, disease, and treatment characteristics

\begin{tabular}{|c|c|c|}
\hline & $N$ or median & $\%$ or range \\
\hline Patients & 46 & \\
\hline \multicolumn{3}{|l|}{ Clinical characteristics } \\
\hline Age at brachytherapy (years) & 49.9 & 31.6-71.4 \\
\hline \multicolumn{3}{|l|}{ Histology } \\
\hline Squamous cell carcinoma & 38 & $82.6 \%$ \\
\hline Adenocarcinoma & 7 & $15.2 \%$ \\
\hline Adenosquamous carcinoma & 1 & $2.2 \%$ \\
\hline Clinical tumor size $(\mathrm{cm})$ & 6 & $2-9$ \\
\hline \multicolumn{3}{|l|}{ FIGO stage (2009) } \\
\hline IB1 & 3 & $6.5 \%$ \\
\hline IB2 & 13 & $28.3 \%$ \\
\hline$\| \mathrm{A} 1$ & 0 & $0.0 \%$ \\
\hline IIA2 & 1 & $2.2 \%$ \\
\hline$\underline{I I B}$ & 20 & $43.5 \%$ \\
\hline IIIB & 7 & $15.2 \%$ \\
\hline IVA & 2 & $4.3 \%$ \\
\hline \multicolumn{3}{|l|}{ Staging MRI } \\
\hline No & 18 & $39.1 \%$ \\
\hline Yes & 28 & $60.9 \%$ \\
\hline \multicolumn{3}{|l|}{ Staging PET } \\
\hline No & 5 & $10.9 \%$ \\
\hline Yes & 41 & $89.1 \%$ \\
\hline \multicolumn{3}{|l|}{ Treatment characteristics } \\
\hline EBRT pelvic dose (Gy) & 45 & $45-50.4$ \\
\hline EBRT fractions & 25 & $25-28$ \\
\hline \multicolumn{3}{|l|}{ EBRT LN boost } \\
\hline No & 22 & $47.8 \%$ \\
\hline Yes & 24 & $52.2 \%$ \\
\hline \multicolumn{3}{|l|}{ EBRT parametrial boost } \\
\hline No & 41 & $89.1 \%$ \\
\hline Yes & 5 & $10.9 \%$ \\
\hline \multicolumn{3}{|l|}{ Tandem length } \\
\hline $4 \mathrm{~cm}$ & 3 & $6.5 \%$ \\
\hline $6 \mathrm{~cm}$ & 41 & $89.1 \%$ \\
\hline $8 \mathrm{~cm}$ & 2 & $4.3 \%$ \\
\hline \multicolumn{3}{|l|}{ Ovoid size (buildup diameter) } \\
\hline Mini $(1.6 \mathrm{~cm})$ & 9 & $19.6 \%$ \\
\hline Small $(2.0 \mathrm{~cm})$ & 28 & $60.9 \%$ \\
\hline Medium $(2.5 \mathrm{~cm})$ & 9 & $19.6 \%$ \\
\hline $\begin{array}{l}\text { Brachytherapy total prescription } \\
\text { dose (Gy) }\end{array}$ & 25 & $25-29$ \\
\hline CTV-D $90 \%$ total EQD 2 & 88.35 & $71.9-118.3$ \\
\hline$\underline{\text { Bladder } \mathrm{D}_{2 \mathrm{cc}} \text { total } E Q D_{2}(\mathrm{~Gy})}$ & 69.85 & $53.0-104.3$ \\
\hline Rectal $D_{2 c c}$ total EQD $(G y)$ & 63.2 & $53.4-75.4$ \\
\hline Sigmoid $\mathrm{D}_{2 \mathrm{cc}}$ total $\mathrm{EQD}_{2}(\mathrm{~Gy})$ & 61.4 & $50.3-82.2$ \\
\hline
\end{tabular}

$C T V$ - clinical target volume, $D_{2 c c}$ - maximum dose received by $2 c c$ of the volume, $D_{90 \%}$ - maximum dose received by $90 \%$ of the volume, EBRT - external beam radi ation therapy, ECOG - Eastern Cooperative Oncology Group performance status, $\mathrm{EOD}_{2}$ - equivalent total dose in 2-Gy fractions (assuming $\alpha / \beta$ of $10 \mathrm{~Gy}$ for tumor and 3 Gy for normal tissues), FIGO - International Federation of Gynecology and Obstetrics, MRI - magnetic resonance imaging, PET - positron emission tomography
Table 2. Matched-pair analysis comparing dosimetric values from CT only based planning to MRI-based planning

\begin{tabular}{lccc} 
& $\begin{array}{c}\text { Fraction 1 } \\
\text { (Gy) } \\
\text { (CT-guided) }\end{array}$ & $\begin{array}{c}\text { Fraction 2-5 } \\
\text { average (Gy) } \\
\text { (MRI-fused) }\end{array}$ & $P$-value \\
\hline $\mathrm{D}_{90 \%}$ HR-CTV & 6.07 & 6.64 & 0.017 \\
\hline $\mathrm{D}_{2 c c}$ rectum & 2.96 & 3.17 & 0.01 \\
\hline $\mathrm{D}_{2 c c}$ bladder & 3.72 & 3.84 & 0.442 \\
\hline $\mathrm{D}_{2 c c}$ sigmoid & 2.76 & 2.87 & 0.29
\end{tabular}

$H R$-CTV - high-risk clinical target volume, $D_{2 c c}$ - maximum dose received by $2 c c$ of the volume, $D_{90 \%}$ - maximum dose received by $90 \%$ of the volume

Average dose to structures

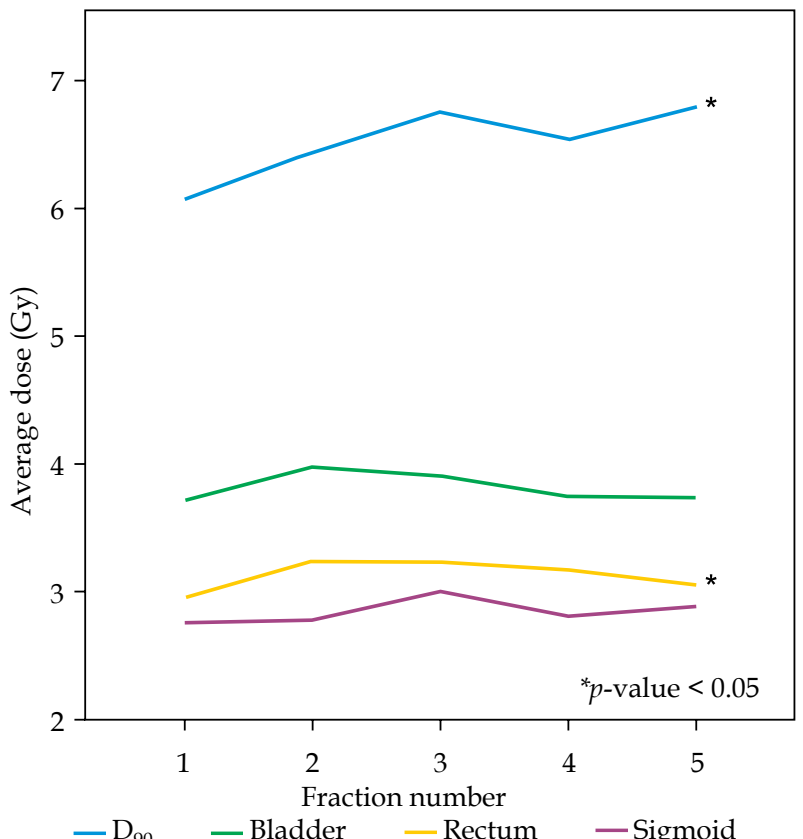

Fig. 2. Mean dose to structures by fraction number for the entire cohort $(n=46)$. Fractions $2-5$ were based on MRI for HR-CTV delineation. $\mathrm{D}_{90}$, maximum dose received by $90 \%$ of the volume to the HR-CTV

HR-CTV change from CT to MRI-based target delineation

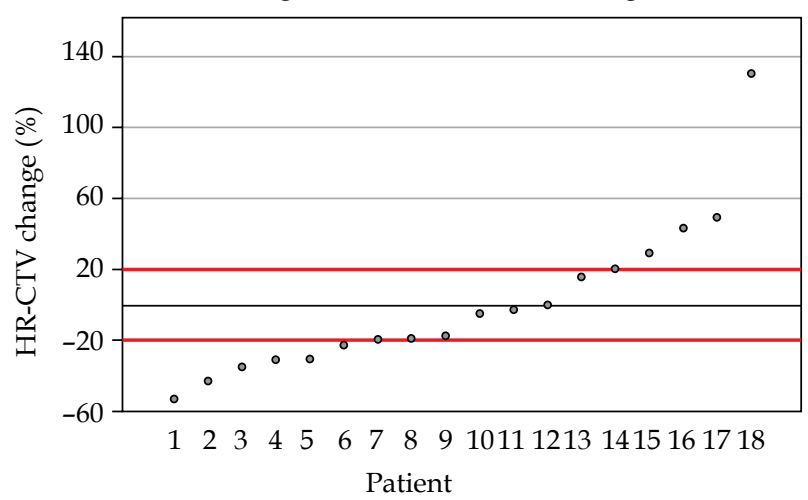

Fig. 3. Percent change from CT to MRI-based target delineation of the HR-CTV for the patients with complete volumetric data $(n=18)$ 
A prospective multicenter study from France reported an improved local control and toxicity with the transition from 2D to 3D planning [8]. Long-term outcomes from prospective studies with MRI-based planning are maturing and promising [9]. Clinical outcomes using our described technique have been previously reported, although is limited by retrospective data and patient heterogeneities [10]. When compared to CT only brachytherapy, the MRI fusion method resulted in fewer late toxicities and equivalent local control. The current study is unique because we evaluated patient-specific volume changes between fractions, as opposed to comparing treatment with or without MRI. Better tumor visualization allows for manual dose optimization techniques theoretically improving therapeutic ratio for each patient [11]. Further optimization of imaging protocols in interstitial setting for locally advanced tumors are worth investigating in future studies.

Our technique and present analysis have limitations. First, MRI is only performed once, and therefore we would not capture ongoing changes during brachytherapy treatment. Second, while a Smit sleeve is in place during MRI and assists with co-registration, tissue deformity by applicators must be accounted for on CT by the treating physician for each fraction. Limitations to this analysis include a small number of patients with full volumetric data, although HR-CTV volume changes appeared as normally distributed in both directions, implying these expected changes with MRI fusion can be applied to a larger cohort. Lastly, a large number of patients with long-term follow-up using this technique is required to validate clinical superiority to CT only based planning.

\section{Conclusions}

The use of asynchronous MRI for target delineation, with co-registration to CT for each fraction of brachytherapy was associated with higher $\mathrm{D}_{90}$ to the HR-CTV. We observed slightly higher $D_{2 c c}$ rectal doses with MRI fusion, but cumulative rectal doses were within accepted thresholds. High-risk target volumes were not consistently increased or decreased, but MRI fusion was associated with target volume changes greater than $20 \%$ in over half of the treated patients.

\section{Disclosure}

The authors report no conflict of interest.

\section{References}

1. Ho CM, Chien TY, Jeng CM et al. Staging of cervical cancer: comparison between magnetic resonance imaging, computed tomography and pelvic examination under anesthesia. J Formos Med Assoc 1992; 91: 982-990.

2. Sullivan T, Yacoub JH, Harkenrider MM et al. Providing MR imaging for cervical cancer brachytherapy: lessons for radiologists. Radiographics 2018; 38: 932-944.

3. Grover S, Harkenrider MM, Cho LP et al. Image guided cervical brachytherapy: 2014 survey of the American Brachytherapy Society. Int J Radiat Oncol Biol Phys 2016; 94: 598-604.

4. Trifiletti DM, Libby B, Feuerlein S et al. Implementing MRIbased target delineation for cervical cancer treatment within a rapid workflow environment for image-guided brachytherapy: A practical approach for centers without in-room MRI. Brachytherapy 2015; 14: 905-909.

5. Han K, Milosevic M, Fyles A et al. Trends in the utilization of brachytherapy in cervical cancer in the United States. Int J Radiat Oncol Biol Phys 2013; 87: 111-119.

6. Dimopoulos JC, Petrow P, Tanderup K et al. Recommendations from Gynaecological (GYN) GEC-ESTRO Working Group (IV): Basic principles and parameters for MR imaging within the frame of image based adaptive cervix cancer brachytherapy. Radiother Oncol 2012; 103: 113-122.

7. Viswanathan AN, Dimopoulos J, Kirisits C et al. Computed tomography versus magnetic resonance imaging-based contouring in cervical cancer brachytherapy: results of a prospective trial and preliminary guidelines for standardized contours. Int J Radiat Oncol Biol Phys 2007; 68: 491-498.

8. Charra-Brunaud C, Harter V, Delannes $M$ et al. Impact of 3D image-based PDR brachytherapy on outcome of patients treated for cervix carcinoma in France: results of the French STIC prospective study. Radiother Oncol 2012; 103: 305-313.

9. Pötter R, Tanderup K, Kirisits C et al. The EMBRACE II study: The outcome and prospect of two decades of evolution within the GEC-ESTRO GYN working group and the EMBRACE studies. Clin Transl Radiat Oncol 2018; 9: 48-60.

10. Dutta SW, Trifiletti DM, Pugh KJ et al. Integration of MRI target delineation into rapid workflow cervical cancer brachytherapy: Impact on clinical outcomes. J Med Imaging Radiat Oncol 2018; 62: 716-725.

11. Mohamed S, Lindegaard JC, de Leeuw AA et al. Vaginal dose de-escalation in image guided adaptive brachytherapy for locally advanced cervical cancer. Radiother Oncol 2016; 120 : 480-485. 